\title{
Factors Associated with Risk Behaviors Towards Hepatitis B Among Migrant Workers: A Cross-sectional Study Based on Theory of Planned Behavior
}

\author{
Hui Xiang \\ Chongqing Medical University \\ Mingjing Li \\ Chongqing Medical University \\ Meng Xiao \\ Peking University \\ Min Liu \\ Chongqing Medical University \\ Xiaoshan Su \\ Chongqing Medical University \\ Dashu Wang \\ Army Medical University \\ Ke Li \\ Beijing Institute of Technology \\ Rui Chen \\ Beijing Institute of Technology \\ Lin Gan \\ Chongqing Medical University \\ Kun Chu \\ Chongqing Medical University \\ Yu Tian \\ Nan'an CDC \\ Xiaojun Tang \\ Chongqing Medical University \\ Xun Lei ( $\square$ leixun521@163.com) \\ Chongqing Medical University
}

Research Article

Keywords: Rural-to-urban migrant worker, hepatitis B, theory of planned behavior, risk behavior, behavioral intention

Posted Date: December 3rd, 2020

DOI: https://doi.org/10.21203/rs.3.rs-118301/v1

License: (9) (i) This work is licensed under a Creative Commons Attribution 4.0 International License. Read Full License 


\section{Abstract}

Background: Rural-to-urban migrant workers are susceptible to hepatitis B (HB) because they lack self-protection awareness and social support. The present study aimed to investigate the current status of risk behaviors (RB) regarding HB among migrant workers and the influencing factors defined by the Theory of Planned Behavior (TPB).

Methods: A cross-sectional study was performed by two-stage cluster sampling from June to December 2018 in Chongqing, China. Logistic regression was adopted to explore factors associated with HB-related RB and behavioral intention (BI).

Results: A total of 1299 migrant workers were recruited in the surveys, among whom $29.56 \%$ respondents have performed HB-related RB and $85.53 \%$ had the BI. $58.19 \%$ of respondents having sexual activities never wore a condom. The risk scores of attitudes towards behavior (AB), subjective norms (SN), experience of behavior (EB) and regret feeling (RF) were positively associated with $\mathrm{BI}$, while the sores of $\mathrm{AB}, \mathrm{EB}$ and $\mathrm{BI}$ were positively associated with $\mathrm{RB}$.

Conclusions: A considerable proportion of migrant workers have had HB-related risk behaviors or had the behavioral intention. Theory-grounded education, focused on the identified TPB variables, may play a significant role in improving the cognition and behaviors towards HB.

\section{Introduction}

Hepatitis B (HB), a leading cause of human's liver cirrhosis and liver cancer, remains a major threat to global public health, particularly in the Asian-Pacific and sub-Saharan Africa regions [1]. There are nearly 2.57 billion hepatitis B surface antigen (HBsAg)-positive people, and $887,000 \mathrm{HB}$ cases dying of liver damage and complications worldwide according to the data from the World Health Organization (WHO) in 2015,the WHO also urged countries to invest in eliminating hepatitis, especially low-and middle-income countries[2].As one of the countries with high $\mathrm{HB}$ endemicity reported by WHO, China has more than 90 million HBpatients and 100,000 annually new HBV infections[2]According to the National Hepatitis Seroepidemiology Survey in 2006,although the (HBsAg)-positive rate has already declined to $2.08 \%$ among Chinese aged from 1 to 14 ,the (HBsAg)-positive rate in populations aged between 15 to 59 was 8.57\% [3]. Nevertheless, the overall (HBsAg)-positive rate was still over 8\% for Chinese adults in 2016 [4].

Hepatitis B virus (HBV)is mostly transmitted through blood or body fluids of infected individuals[4], and having sex contact and using contaminated injector are known as the major routes for HBV infection among adults $[5,6]$. Therefore, risk behaviors related to HB refer to unprotected sex, whoring, multi-partner sex, sharing injectors for drug use and so on[6, 7].

Rural-to-urban migrant workers, almost accounting for the whole of internal migrant population in China, were commonly defined as people who left rural area where they used to live for towns or cities to seek better employment opportunities and higher-incomes[8].The latest Migrant Workers Monitoring Investigation Report of China showed that the number of migrant workers has increased to 288.36 million by 2018[9]. Most migrant workers are basically low educated, engage in low-incomes and low-skilled jobs, and most of them live a stressful life [10]. They are mostly at sexually active ages but commonly being single or away from their spouses[10]. And the majority of them have poor sex-related to knowledge and little self-protection awareness, insufficient social support and limited access to health care[8]. Therefore, migrant workers are more likely to have HB-related behavior mentioned above, in which case the risk of HBV infection will be increased among them. Previous surveys showed that construction workers had unprotected sex, commercial sex, casual sex, blood selling and drug use, accounting for $14.9 \%, 7.9 \%, 8.4 \%$ and $0.7 \%$ respectively[11, 12$]$ And previous studies also found that migrant workers had relatively higher susceptibility to HBV infection compared with non-migrants and local dwellers[13].As summarized in a systematic review and meta-analysis by Zou et.al, the prevalence of viral hepatitis among rural to urban migrant workers was $0.45 \%$ and 38.5 higher odds of infection than general populations in China[13].In addition, the frequent flow of migrant workers is possible to enlarge HBV spread to general population facilitate the regional transmission across China [14].It is therefore important to know the status of HB-related risk behaviors of migrant workers and have insight into the determinants.

One of the most recognized theories to understand behaviors is the theory of planned behavior (TPB, Ajzen, 1991). TPB states three conceptual modules determine behavior:(1)attitude toward the behavior (AB), refers to favorable/unfavorable appraisal of the behavior;(2) subjective norms (SN), refers to perception of social pressure to perform/not perform the behavior;(3) perceived behavioral control (PBC), refers to the perceived ease or difficulty to perform the behavior. TPB postulates that $A B, S N$, and PBC lead to the formation of a behavioral intention. The more favorable the $A B$ and $S N$ are, and the greater the $\mathrm{PBC}$ is, the stronger would be a person's intention to perform the behavior in question, and the higher possibility that the person would go into action[15, 16]In addition, Previous studies also argued that some independent variables, like experience and/or regret of performing a behavior, would directly or indirectly influence the behavioral intention and worth being taken into account to improve the TPB framework[17, 18]. To address the issue of health behaviors, TPB variables has been widely adopted to interpret HIV/AIDS-related behaviors, particularly for the highly susceptible groups such as commercial sex workers and men who have sex with men (MSM) [19, 20] And previously TPB was also applied to explore determinants on smoking, drinking and health-seeking behaviors of people $[21,22]$. Nevertheless, the study that examined HB-related risk behaviors of rural-to-urban migrants was rarely reported.

The present study was the first attempt to (1) understand the status of risk behaviors related to HB that performed by migrant workers and (2) detect what and how the factors motivate and influence the behavioral intention and practical behaviors on the basis of TPB theory.

\section{Methods}

\section{Study sites and sampling}

Chongqing, located in southwestern China, is the largest municipality directly under the Chinese central government. It is regarded as "miniature China" because its geographic characteristics, urban-rural distribution and social-economic profile are close to the national average[23]. The city area of Chongqing, 
one of the busiest places Chinese migrant workers keep flowing in, consists of nine administrative districts with an area of $5472.82 \mathrm{Km}^{2}$ and a population of 8.65 million, among which immigrants take about $23.5 \%[23,24]$.The (HBsAg)-positive rate among migrant workers in the city area of Chongqing was $8.6 \%$ [25].And there were nearly 26,000 new infections of viral hepatitis in 2016 in terms of the Health Statistic Yearbook of Chongqing [24].

Two-stage stratified cluster sampling was performed to recruit participants between June 2018 and January2019. Firstly, nine districts of Chongqing's city area were categorized into three stratifications-more developed, medium developed and less developed-by economic development, geographic background and population density, and then three districts were randomly selected to represent for each stratification respectively. Secondly, two enterprises were purposively sampled in each district, including the manufacturing, construction, wholesale and retail industry, transportation industry, hotel and catering industry and community services. Local Center for Disease Control and Prevention, Health Supervision Institute, and Urban-rural Development Committee helped to coordinate with sampled units. The inclusion criteria of target individuals were (1) 18 years and above, (2) having been in the Chongqing's city area for at least six months, (3) not registered as Chongqing urban resident, (4) engaging mainly in the secondary or tertiary industry. Given a considerable part of migrant workers were low-educated or illiterate, trained investigators assisted to explain the questions in mandarin and Chongqing dialect. Subjects who were unable to understand the questionnaire items or refused to be surveyed were excluded. Participants were reassured that all responses would be anonymous and written informed consent was granted by each respondent. Surveys were conducted in relatively undisturbed environment and peak working hours were avoided to guarantee the quality of surveys. Completeness of each questionnaire was double-checked by investigators.

\section{Study Instrument}

The questionnaire was constructed basically in terms of the TPB on health-related to behaviors and perceptions in published studies[19, 20]. Experts in epidemiology and hepatology were involved to modify the logic and wordings of the questionnaire. A pilot survey was conducted with 90 migrant workers in nearby restaurants and construction sites. The final version consisted of nine modules with Cronbach's Alpha coefficients ranging from 0.759 to 0.968 , and confirmatory factor analysis (CFA) showed a good fitting degree $\left(X^{2} / \mathrm{df}=1.859, \mathrm{RMSEA}=0.039, \mathrm{GFI}=0.900, \mathrm{AGFI}=0.883, \mathrm{CFI}=0.969, \mathrm{IFI}=0.969\right)$. TPB variables was assessed using a five-point semantic differential scale, and the average item score for each module was computed to be used as the scale score. The higher the scores are, the more risky the respondents would be. The definition of each modules and variable scales were shown in Table 1.The items of different dimensions and positive and negative items were sorted at intervals to prevent the respondents from having a Socially Desirable Responding or picking an initial scale for each item. 
Table 1

Definition and item scales of each module in the questionnaire for HB-related behaviors

\begin{tabular}{|c|c|c|}
\hline Modules & Definition & Variable scales \\
\hline $\begin{array}{l}\text { Social } \\
\text { demographic }\end{array}$ & $\begin{array}{l}\text { Respondents were asked about gender, age, hometown, ethnicity, education } \\
\text { background, marital status, cohabitation with spouse/partner, accommodation } \\
\text { condition, years of being a migrant worker, type of work, job position, working hours per } \\
\text { day, monthly personal income, whether send money to the family, whether smoke, } \\
\text { whether drink, HB vaccination behavior, willingness of vaccination against HB (only for } \\
\text { those who was not vaccinated yet). }\end{array}$ & - \\
\hline HB knowledge & $\begin{array}{l}\text { Knowledge level involves questions of individual's understanding on transmitted routes } \\
\text { and preventive measures towards HB }\end{array}$ & $\begin{array}{l}\text { Each correct response of knowledge on } \\
\text { HB was scored as one point while } \\
\text { incorrect response or unknown value zero. }\end{array}$ \\
\hline \multicolumn{3}{|l|}{ TPB } \\
\hline $\begin{array}{l}\text { HB-relatedrisk } \\
\text { behaviors (RB) }\end{array}$ & $\begin{array}{l}\text { Unprotected sexual behaviors (never or rarely wear a condom), casual sexual behavior, } \\
\text { commercial sexual behavior, homosexual behavior, IDUs, blood selling /transfusion } \\
\text { illegally, and sharing toothbrushes/towels. }\end{array}$ & $\begin{array}{l}\text { RB questions were valued by 5-point } \\
\text { semanticdifferential scale: from "never" } \\
\text { (1) to "often" (5). An example is "Have you } \\
\text { had casual sex in the last six months?" }\end{array}$ \\
\hline $\begin{array}{l}\text { Behavioral } \\
\text { intention (BI) }\end{array}$ & BI refers to person's readiness to perform a given behavior. & $\begin{array}{l}\text { BI questions were measured by 5-point } \\
\text { semantic differential scale: } \\
\text { from"absolutely impossible"(1)to } \\
\text { "absolutely possible"(5).An example is: "Is } \\
\text { it possible for you to have commercial } \\
\text { sexual behavior?" }\end{array}$ \\
\hline $\begin{array}{l}\text { Attitudes toward } \\
\text { a behavior (AB) }\end{array}$ & $\begin{array}{l}A B \text { refers to the degree to which performance of the risk behavior is positively or } \\
\text { negatively valued. }\end{array}$ & $\begin{array}{l}\text { AB questions were measured by 5-point } \\
\text { semantic differential scale: from "very } \\
\text { much unsafe" }(1) \text { to"very much safe" }(5) \text {. } \\
\text { An example is: "Do you think it is safe to } \\
\text { have commercial sex?" }\end{array}$ \\
\hline $\begin{array}{l}\text { Subjectivenorms } \\
\text { (SN) }\end{array}$ & $\begin{array}{l}\mathrm{SN} \text { refers the perceptions of social pressure to engage or not to engage in risk } \\
\text { behaviors. }\end{array}$ & $\begin{array}{l}\text { SN questions were measured by5- } \\
\text { ponitsemantic differential scale: } \\
\text { from"strongly agree" }(1) \text { to "strongly } \\
\text { disagree" }(5) \text {. An example is: "Do you } \\
\text { agree with your friends if they advise you } \\
\text { not to actany of the above behaviors?" }\end{array}$ \\
\hline $\begin{array}{l}\text { Perceived } \\
\text { behavioral } \\
\text { control(PBC) }\end{array}$ & PBC refers a person feels she/he is in control of a given risk behaviors. & $\begin{array}{l}\text { PBC questions were measured by 5-point } \\
\text { semantic } \\
\text { differentialscale:from"verymuchable"( } 1) \\
\text { "very much unable"(5). An example is: } \\
\text { "Are you able to decide whether or not } \\
\text { have casual sex by yourself?" }\end{array}$ \\
\hline $\begin{array}{l}\text { Experience of } \\
\text { behavior (EB) }\end{array}$ & EB was referred the safe or unsafe perception towards related to risk behaviors. & $\begin{array}{l}\text { EB questions were measured by 5-point } \\
\text { semantic differential scale: from } \\
\text { stronglydisagree(1)tostrongly agree(5). An } \\
\text { example is: "I had behaviors mentioned } \\
\text { above to meet physiological needs." }\end{array}$ \\
\hline $\begin{array}{l}\text { Regret feeling } \\
\text { (RF) }\end{array}$ & $\begin{array}{l}\text { RF refers to an individual's psychological regret and shame on HB risk behaviors that } \\
\text { he ever did and/or he plans to have. And RF includes retrospective regretand } \\
\text { prospective regret. }\end{array}$ & $\begin{array}{l}\text { RF questions were measured by 5-point } \\
\text { semantic differential scale: from "very } \\
\text { much"(1) to"not at all"(5). Examplesare: } \\
\text { "Did you regret after having commercial } \\
\text { sex behavior?" and "Will you regret if you } \\
\text { had commercial sex?" }\end{array}$ \\
\hline
\end{tabular}

\section{Data analysis}

Survey data were double-checked and entered into a database by Epidata 3.1 (The EpiData Association, Odense, Denmark).All data were analyzed using IBM SPSS 22.0 (SPSS Institute, Chicago, USA). Categorical data were assessed by the number and proportion of responders. Continuous variables of sociodemographics, such as age, years of being a migrant worker, working hours per day, were converted into categorical variables and then described by number and proportion of responders. Knowledge levels of responders were divided into poor, medium and good by scores less than 7, $8 \sim 10,11 \sim 13$ respectively [26]. TPB variable were involved as continuous variable with average scores. With regard to the association analyses, independent variables were identified as the variables in the modules of socio-demographics, HB knowledge and TPB framework, and the dependent variables were identified as behavioral intention and HB-related risk behaviors. For the dependent variables, behavioral intention was dichotomized into "never had an intent" and "had an intent for at least a behavior" and HB-related risk behaviors were dichotomized into "never had risk behavior" and "had at least once risk behavior". Univariate analyses were performed with independent variables of socio-demographic and HB knowledge with the two dependent variables by Chi-square tests. And independent variables with $P$-values less than 0.10 in the univariate analyses were subsequently inputted into the logistic regression models $(a=0.05, \beta=0.10)$ along with variables in TPB modules to detect the possible influence factors for the two dependent variables. Binary logistic regressions were fitted with the dependent variables by entering three blocks of variables: block I, socio-demographics and knowledge level; block II, TPB variables; block III, demographics, knowledge level and TPB variables. BI was not included in the block II and block III when it was regarded as dependent variable. Dummy variables were coded for 
variables with more than two values, and variables stepwise entered into the models. Adjusted odds ratios (ORs) and $95 \%$ confidence intervals (Cls) were computed, and $P$-values less than 0.05 were deemed statistically significant.

\section{Results}

\section{Basic characteristics}

A total of 1528 migrant workers meeting the eligible criteria were recruited.1299 (85.02\%) respondents completed the questionnaire, among whom758(58.35\%) were females. The median age of respondentswas30.58 \pm 21.18 years, ranging from 18 to 68.901 (69.36\%) respondents were married or had a relationship, but $670(51.58 \%)$ respondents were not living with their spouse/partner. Meanwhile,43(26.33\%) were single and 55(4.23\%) were divorced/windowed. There were $443(34.10 \%)$ respondents having a monthly income above 4000 RMB and 451 (43.53\%) regularly sending money back home. 626 (48.19\%) respondents had an education background of junior school or below, 246(18.93\%) respondents drank alcohol, and 921(70.90\%) respondents showed a poor HB knowledge level. The top three accesses for the migrant workers to obtain HB knowledge and information were hearing from friends/family members (53.88\%), followed by television or radio (38.12\%) and internet or mobile phone (27.95\%) respectively (Table 3).

\section{HB behavior intention}

$1111(85.52 \%$ ) respondents ever had an intention to have risk behaviors in the last six months (Table 2 ). Respondents who have had an intention to perform casual sex, commercial sex, homosexual/anal sex, take drugs through sharing injectors, sell/transfuse blood illegally and share towels/toothbrushes were $55.65 \%, 36.26 \%, 73.90 \%, 26.10 \%, 31.18 \%$ and $53.50 \%$ respectively. As for condom use, more than half of the respondents $(62.36 \%)$ were very willing to wear a condom when having HB risk sexual behaviors (Table 4). 
Table 2

Characteristic differences inHB-related behaviors and behavioral intention

\begin{tabular}{|c|c|c|c|c|c|c|c|c|c|}
\hline \multirow[t]{2}{*}{ Variables } & \multirow[b]{2}{*}{ Total } & \multicolumn{4}{|c|}{ Risk behavioral intention } & \multicolumn{4}{|c|}{ Risk behavior } \\
\hline & & $\begin{array}{l}\text { Non-risk behavioral } \\
\text { intention group }\end{array}$ & $\begin{array}{l}\text { Risk behavioral } \\
\text { intention group }\end{array}$ & $\chi^{2}$ & $P$-value & $\begin{array}{l}\text { Non-risk } \\
\text { behavior } \\
\text { group }\end{array}$ & $\begin{array}{l}\text { Risk } \\
\text { behavior } \\
\text { group }\end{array}$ & $\chi^{2}$ & $P$-value \\
\hline \multicolumn{10}{|l|}{ Gender } \\
\hline Male & 541 & $51(9.43)$ & $490(90.57)$ & 19.07 & $<0.001$ & $350(64.70)$ & 191(35.30) & 14.69 & $<0.001$ \\
\hline Female & 758 & 137(18.07) & 621(81.93) & & & $565(74.54)$ & $193(25.46)$ & & \\
\hline \multicolumn{10}{|l|}{ Age group } \\
\hline $18 \sim 30$ & 632 & $64(10.13)$ & $568(89.87)$ & 22.52 & $<0.001$ & $419(66.30)$ & $213(33.70)$ & 10.78 & 0.013 \\
\hline $31 \sim 40$ & 252 & $39(15.48)$ & 213(84.52) & & & 191(75.79) & $61(24.21)$ & & \\
\hline $41 \sim 50$ & 276 & $54(19.57)$ & 222(19.57) & & & $205(74.28)$ & $71(25.72)$ & & \\
\hline $51 \sim$ & 139 & $31(22.30)$ & 108(77.70) & & & $100(71.94)$ & $39(28.06)$ & & \\
\hline \multicolumn{10}{|l|}{ Hometown } \\
\hline Rural area in Chongqing & 964 & 136(14.11) & $828(85.89)$ & 0.40 & 0.526 & $671(69.61)$ & 293(30.39) & 1.25 & 0.264 \\
\hline $\begin{array}{l}\text { Rural area in other } \\
\text { cities/provinces }\end{array}$ & 335 & $52(15.52)$ & 283(84.48) & & & $244(72.84)$ & $91(27.16)$ & & \\
\hline \multicolumn{10}{|l|}{ Ethnicity } \\
\hline Han & 1226 & $180(14.68)$ & 1046(85.32) & 0.77 & 0.380 & $865(70.55)$ & $361(29.45)$ & 0.14 & 0.708 \\
\hline Others & 73 & $8(10.96)$ & 65(89.04) & & & $50(68.49)$ & $23(31.51)$ & & \\
\hline \multicolumn{10}{|l|}{ Education background } \\
\hline Primary school or below & 153 & $33(21.57)$ & $120(78.43)$ & 10.53 & 0.015 & $110(71.90)$ & $43(28.10)$ & 4.10 & 0.251 \\
\hline Junior middle school & 473 & $59(12.47)$ & $414(87.53)$ & & & $346(73.15)$ & $127(26.85)$ & & \\
\hline High school & 476 & $61(12.82)$ & $415(87.18)$ & & & $329(69.12)$ & $147(30.88)$ & & \\
\hline College and above & 197 & $35(17.77)$ & $162(82.23)$ & & & $130(65.99)$ & $67(34.01)$ & & \\
\hline \multicolumn{10}{|l|}{ Marital status } \\
\hline Single & 343 & $39(20.74)$ & $304(27.36)$ & 3.65 & 0.161 & $248(72.30)$ & $95(27.70)$ & 3.45 & 0.178 \\
\hline Married/having a partner & 901 & $140(15.54)$ & $761(84.46)$ & & & 634(70.37) & $267(29.63)$ & & \\
\hline Divorced/widowed & 55 & $9(16.36)$ & $46(83.64)$ & & & $33(60.00)$ & $22(40.00)$ & & \\
\hline \multicolumn{10}{|l|}{ Live with Spouse/Partner } \\
\hline No & 670 & $112(16.72)$ & $558(83.28)$ & 5.63 & 0.017 & $465(69.40)$ & $205(30.60)$ & 0.71 & 0.399 \\
\hline Yes & 629 & $76(12.08)$ & $553(87.92)$ & & & $450(71.54)$ & $179(28.46)$ & & \\
\hline \multicolumn{10}{|l|}{ Accommodation } \\
\hline $\begin{array}{l}\text { Self-renting room/ Self- } \\
\text { purchased house }\end{array}$ & 767 & $126(16.43)$ & $641(83.57)$ & 5.78 & 0.016 & $542(70.66)$ & $225(29.34)$ & 0.05 & 0.830 \\
\hline Co-renting room/ Dormitory & 532 & $62(11.65)$ & $470(88.35)$ & & & $373(70.11)$ & $159(29.89)$ & & \\
\hline \multicolumn{10}{|l|}{$\begin{array}{l}\text { Years of being a migrant } \\
\text { worker }\end{array}$} \\
\hline Six months -three years & 486 & $71(14.61)$ & 415(85.39) & 1.21 & 0.547 & $346(71.19)$ & $140(28.81)$ & 0.22 & 0.897 \\
\hline Three years-six years & 265 & $33(12.45)$ & $232(87.55)$ & & & $185(69.81)$ & $80(30.19)$ & & \\
\hline More than six years & 548 & $84(15.33)$ & $464(84.67)$ & & & $384(70.07)$ & 164(29.93) & & \\
\hline \multicolumn{10}{|l|}{ Type of work } \\
\hline \multicolumn{10}{|c|}{ asecondary industry includes manufacturing industry and construction industry; } \\
\hline \multicolumn{10}{|c|}{ bTertiary Industry includes catering industry, hotel attendant, logistics industry, wholesale / retail business and part-time jobs. } \\
\hline
\end{tabular}




\begin{tabular}{|c|c|c|c|c|c|c|c|c|c|}
\hline \multirow{2}{*}{$\begin{array}{l}\text { Variables } \\
\text { Secondary industry a }\end{array}$} & \multirow[b]{2}{*}{584} & \multicolumn{4}{|c|}{ Risk behavioral intention } & \multicolumn{4}{|c|}{ Risk behavior } \\
\hline & & $71(12.16)$ & $513(87.84)$ & 4.59 & 0.032 & 407(69.69) & 177(30.31) & 0.28 & 0.594 \\
\hline Tertiary Industry ${ }^{\mathbf{b}}$ & 715 & 117(16.36) & $598(83.64)$ & & & 508(71.05) & 207(28.95) & & \\
\hline \multicolumn{10}{|l|}{ Job position } \\
\hline Ordinary employee & 1076 & 152(14.13) & $924(85.87)$ & 0.61 & 0.436 & 766(71.19) & $310(28.81)$ & 1.70 & 0.193 \\
\hline Group leader/ Administrator & 223 & $36(16.14)$ & 187(83.86) & & & $149(66.82)$ & 74(33.18) & & \\
\hline \multicolumn{10}{|l|}{ Working hours per day } \\
\hline$\leq 8 \mathrm{~h}$ & 384 & 72(18.75) & $312(81.25)$ & 8.06 & 0.005 & $287(74.74)$ & 9725.26) & 4.84 & 0.028 \\
\hline$>8 \mathrm{~h}$ & 915 & 116(12.68) & 799(87.32) & & & $628(68.63$ & 287(31.37) & & \\
\hline \multicolumn{10}{|l|}{$\begin{array}{l}\text { Monthly personal income } \\
\text { (RMB) }\end{array}$} \\
\hline$<2500$ & 355 & $71(20.00)$ & $284(80.00)$ & 12.06 & 0.002 & 26775.21) & $88(24.79)$ & 6.68 & 0.035 \\
\hline $2501 \sim 4000$ & 501 & $62(12.38)$ & $439(87.62)$ & & & $352(70.26)$ & 149(29.74) & & \\
\hline$>4000$ & 443 & $55(12.42)$ & $388(87.58)$ & & & 296(66.82) & 147(33.18) & & \\
\hline \multicolumn{10}{|l|}{$\begin{array}{l}\text { Do you regularly send } \\
\text { money to your family? }\end{array}$} \\
\hline No & 907 & $129(14.22)$ & 778(85.78) & 0.15 & 0.697 & 632(69.68) & $275(30.32)$ & 0.83 & 0.362 \\
\hline Yes & 392 & $59(15.05)$ & $333(84.95)$ & & & 283(72.19) & 109(27.81) & & \\
\hline \multicolumn{10}{|l|}{ Do you smoke? } \\
\hline No & 1008 & 164(16.27) & $844(83.73)$ & 11.74 & $<0.001$ & $741(73.51)$ & $267(26.49)$ & 20.41 & $<0.001$ \\
\hline Yes & 291 & $24(8.25)$ & 267(91.75) & & & 174(59.79) & 117(40.21) & & \\
\hline \multicolumn{10}{|l|}{ Do you drink? } \\
\hline No & 1053 & 167(15.86) & $886(84.14)$ & 8.64 & 0.003 & $771(73.22)$ & 282(26.78) & 20.65 & $<0.001$ \\
\hline Yes & 246 & $21(8.54)$ & 225(91.46) & & & 144(58.54) & 102(41.46) & & \\
\hline \multicolumn{10}{|l|}{ Level of HB knowledge } \\
\hline Poor $(0 \sim 7)$ & 921 & 127(13.79) & 794(86.21) & 10.93 & 0.004 & 648(70.36) & $273(29.64)$ & 0.38 & 0.824 \\
\hline Medium (8 10) & 305 & $41(13.40)$ & 265(86.60) & & & 214(69.93) & $92(30.07)$ & & \\
\hline Good (11 13) & 72 & $20(27.78)$ & $52(72.22)$ & & & $53(73.61)$ & 19(26.39) & & \\
\hline \multicolumn{10}{|l|}{ HaveinoculatedHB vaccine } \\
\hline No & 647 & $86(13.29)$ & $561(86.71)$ & 1.45 & 0.228 & $451(69.71)$ & 196(30.29) & 0.33 & 0.564 \\
\hline Yes & 652 & 102(15.64) & $550(84.36)$ & & & 464(71.17) & 188(28.83) & & \\
\hline \multicolumn{10}{|l|}{$\begin{array}{l}\text { Willing to inoculate HB } \\
\text { vaccine }(N=647)^{c}\end{array}$} \\
\hline No & 398 & $57(14.32)$ & $341(85.68)$ & 0.68 & 0.410 & 285(71.61) & 113(28.39) & 1.50 & 0.221 \\
\hline Yes & 249 & $30(12.05)$ & 219(87.95) & & & $167(67.07)$ & 82(32.93) & & \\
\hline \multicolumn{10}{|c|}{ aSecondary industry includes manufacturing industry and construction industry; } \\
\hline${ }^{\mathrm{b}}$ Tertiary Industry includes ca & ing inc & stry, hotel att & istics indust & ale /ret & busines & and part-time & & & \\
\hline
\end{tabular}


Table 3

Access to hepatitis $B(H B)$ knowledge $(N=1299)$

\begin{tabular}{|ll|}
\hline Source of Obtaining HB Knowledge & $\mathbf{N}(\%)$ \\
\hline Friends or family members & $848(53.88 \%)$ \\
\hline Television or radio & $600(38.12 \%)$ \\
\hline Internet or cell phone APPs & $440(27.95 \%)$ \\
\hline Newspaper or magazine & $310(19.70 \%)$ \\
\hline Doctors & $304(19.31 \%)$ \\
\hline Brochure or booklets & $296(18.81 \%)$ \\
\hline Advertisement & $172(10.93 \%)$ \\
\hline Health education or professional training & $133(8.45 \%)$ \\
\hline
\end{tabular}




\section{Variables \\ Are you possible to have casual sex with people who are not your spouse/partner}

Absolutely impossible

$576 \quad 44.34$

It depends/Little possible

$584 \quad 44.96$

Possible/Absolutely possible

$139 \quad 10.70$

Are you possible to have commercial sex

Absolutely impossible

It depends/Little possible

$421 \quad 32.41$

Possible/Absolutely possible

Are you possible to have homosexual/anal sex

Absolutely impossible

$960 \quad 73.90$

It depends/Little possible

$310 \quad 23.86$

Possible/Absolutely possible

$29 \quad 2.24$

Are you willing to wear a condom when having sex?

Very willing

$810 \quad 62.36$

Willing /Neutral

$293 \quad 22.55$

Unwilling/Absolutely unwilling

$196 \quad 15.09$

Are you possible to share the injector for intravenous drug use

Absolutely impossible

$1072 \quad 82.53$

It depends/Little possible

$215 \quad 16.55$

Possible/Absolutely possible

$\begin{array}{ll}12 & 0.92\end{array}$

Are you possible to sell or transfuse blood illegally

Absolutely impossible

$894 \quad 68.82$

It depends/Little possible

Possible/Absolutely possible

$54 \quad 4.16$

Are you possible to share toothbrushes/towels with others

Absolutely impossible

$604 \quad 46.50$

It depends/Little possible

521

40.10

Possible/Absolutely possible

174

13.40

Have you had casual sex with people who are not your spouse/partner in the last six months

Never

$1166 \quad 89.76$

Rarely/seldom ${ }^{a}$

$108 \quad 8.32$

Sometimes / often

Have you had commercial sex in the last six months

Never

$1238 \quad 95.30$

Rarely/ seldom

$55 \quad 4.24$

Sometimes / often

6

Have you had homosexual behaviors in the last six months

Never

a rarely-less than once per month; seldom-about twice per month; sometimes-about eight timesper month; often-more than twelve time per month

${ }^{b}$ multiple options, the sum of percentages of the options may be not equal to $100 \%$. 


\begin{tabular}{|c|c|c|}
\hline Variables & $\mathbf{N}$ & $\%$ \\
\hline Sometimes / often & 4 & 0.03 \\
\hline \multicolumn{3}{|c|}{ Have you worn a condom when you were having sex $(N=842)$} \\
\hline Never & 490 & 58.19 \\
\hline Sometimes/about half time & 68 & 8.08 \\
\hline Frequently/every time & 284 & 33.73 \\
\hline \multicolumn{3}{|l|}{ Reasons for never using condom $(\mathrm{N}=490)$ * } \\
\hline Have taken other methods of contraception & 210 & 42.60 \\
\hline Uncomfortable to wear a condom & 167 & 33.87 \\
\hline The partner did not ask & 50 & 10.20 \\
\hline Trust in each other & 49 & 9.96 \\
\hline Embarrassing if purchase condom & 43 & 8.74 \\
\hline Do not know how to use condoms & 38 & 7.72 \\
\hline Forgot to use & 29 & 5.89 \\
\hline Too expensive & 9 & 1.83 \\
\hline \multicolumn{3}{|c|}{ Have you sharedtheinjector for intravenous drug use in the last six months } \\
\hline Never & 1286 & 99.00 \\
\hline Rarely/ seldom & 11 & 0.85 \\
\hline Sometimes / often & 2 & 0.15 \\
\hline \multicolumn{3}{|l|}{ Reasons for sharing the injector $(\mathrm{N}=13)^{\mathrm{b}}$} \\
\hline Difficult to get new injector & 7 & - \\
\hline For saving money & 4 & - \\
\hline Could' not refuse the request of others & 2 & - \\
\hline Increase friendship and belongingness & 1 & - \\
\hline \multicolumn{3}{|c|}{ Have yousoldortransfusedblood at illegal clinics in the last six months } \\
\hline Never & 1283 & 98.77 \\
\hline Rarely/ seldom & 13 & 1.00 \\
\hline Sometimes / often & 3 & 0.23 \\
\hline \multicolumn{3}{|c|}{ Reasons for selling or transfusing blood illegally $(N=16)^{b}$} \\
\hline Too expensive to go to a regular hospital & 7 & - \\
\hline More convenient & 7 & - \\
\hline For making money & 2 & - \\
\hline \multicolumn{3}{|c|}{ Have you shared toothbrushes/towels in the last six moths } \\
\hline Never & 1034 & 79.60 \\
\hline Rarely/ seldom & 200 & 15.40 \\
\hline Sometimes / often & 65 & 5.00 \\
\hline \multicolumn{3}{|c|}{ Reasons for sharing toothbrush or towel $(\mathrm{N}=265)^{b}$} \\
\hline Intimate relationship & 156 & 58.87 \\
\hline Took other's by mistake & 93 & 35.09 \\
\hline Did not mind & 38 & 14.34 \\
\hline
\end{tabular}

${ }^{a}$ rarely-less than once per month; seldom-about twice per month; sometimes-about eight timesper month; often-more than twelve time per month ${ }^{b}$ multiple options, the sum of percentages of the options may be not equal to $100 \%$. 


\begin{tabular}{|l|}
\hline Variables \\
\hline For saving money \\
\hline a rarely-less than once per month; seldom-about twice per month; sometimes-about eight timesper month; often-more than twelve time per month \\
\hline b $m$ multiple options, the sum of percentages of the options may be not equal to $100 \%$. \\
\hline
\end{tabular}

\section{HB risk behavior status}

Table 2depictsthat 384(29.56\%) migrant workers have performed HB-related risk behaviors in the last six months. Of all the respondents, 133 (10.24\%) have had causal sexual behavior, $61(4.7 \%)$ have had commercial sex, 40 (3.00\%) have had homosexual/anal sex in the last six months. Among the 842 respondents having sexual behaviors, more than a half (58.19\%) never worn a condom. To explore the reason why not using a condom, $210(42.60 \%)$ respondents indicated that they had other methods of contraception, $167(33.87 \%)$ respondents reported that it was uncomfortable to wear a condom, and $43(8.74 \%)$ respondents felt embarrassed to purchase the condom. There were $265(20.40 \%)$ respondents having shared toothbrushes or towels with friends or family members, and13 (1.00\%) respondents have shared injectors for intravenous drug use and $16(1.23 \%)$ respondents havesold or transfused blood illegally(Table 4).

\section{Influencing factors associated with behavioral intention}

As table 2shows, univariate analyses indicated that the behavioral intention of respondents was significantly different with variables of gender, age, education background, living with spouse/partners, type of accommodation, type of work, working hours per day, monthly personal income, smoking, alcohol drinking and level of HB knowledge respectively $(P<0.05)$. Binary logistic regression detected that migrant workers with an education level of junior middle school ( $\mathrm{OR}=2.16,95 \% \mathrm{Cl}: 1.25 \sim 3.73)$, aged from 18 to 30 ( $\mathrm{OR}=3.49,95 \% \mathrm{Cl}: 1.91 \sim 6.39)$ and from 31 to $40(\mathrm{OR}=2.06,95 \% \mathrm{Cl}$ : $1.13 \sim 3.77)$, were more intent to have HB-related behaviors, while thosebeing female ( $\mathrm{OR}=0.61,95 \% \mathrm{Cl}: 0.39 \sim 0.95)$ were less likely to have the behavioral intention in the block $\nabla$. The scores of $\mathrm{AB}(\mathrm{OR}=9.36,95 \% \mathrm{Cl}: 5.32 \sim 16.46)$, SN (OR = 2.20, 95\% Cl: $1.54 \sim 3.17)$, $\mathrm{EB}(\mathrm{OR}=1.92,95 \% \mathrm{Cl}: 1.43 \sim 2.58)$ and $\mathrm{RF}(\mathrm{OR}=1.20,95 \% \mathrm{Cl}: 1.05 \sim 1.38)$ modules had positive associations with the behavior intention for HB-related risk behaviorsin the block II. In the Block $\nabla$, migrant workers were younger(OR = 2.77, 95\% Cl: $1.41 \sim 5.43)$ and with the poor level of $\mathrm{HB}$ knowledge(OR $=2.10,95 \% \mathrm{Cl}: 1.03 \sim 4.28)$ were more intent to have risk $\mathrm{HB}$ behavior, and the scores of $\mathrm{AB}(\mathrm{OR}=9.49,95 \% \mathrm{Cl}: 5.32 \sim 16.91), \mathrm{SN}(\mathrm{OR}=2.06,95 \% \mathrm{Cl}: 1.44 \sim 2.95), \mathrm{EB}(\mathrm{OR}=2.17,95 \% \mathrm{Cl}: 1.60 \sim 2.94)$ and $\mathrm{RF}(\mathrm{OR}=1.23,95 \% \mathrm{Cl}: 1.06 \sim 1.42)$ remainedto be positivelyassociated with the behavioral intention(Table 5). 
Table 5

Factors associated with intention towards HB-related behaviors and behaviors among migrant workers

\begin{tabular}{|c|c|c|c|c|c|c|c|c|c|c|c|c|}
\hline \multirow[t]{3}{*}{ Variables } & \multicolumn{4}{|c|}{ Intention towards HB-related behaviors } & \multicolumn{7}{|c|}{ HB-related behaviors } & \multirow[b]{2}{*}{ Block $\rrbracket$} \\
\hline & & Block \ & & Block \ & & Block \ & & Block 》 & & 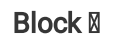 & & \\
\hline & $\begin{array}{l}\text { OR } \\
\text { a }\end{array}$ & $95 \% \mathrm{Cl}^{\mathrm{b}}$ & $\begin{array}{l}\text { OR } \\
a\end{array}$ & $95 \% \mathrm{Cl}^{\mathrm{b}}$ & OR & $95 \% \mathrm{Cl}^{\mathrm{a}}$ & $\begin{array}{l}\text { OR } \\
a\end{array}$ & $95 \% \mathrm{Cl}^{\mathrm{b}}$ & $\begin{array}{l}\text { OR } \\
a\end{array}$ & $95 \% \mathrm{Cl}^{\mathrm{b}}$ & OR & $95 \% \mathrm{Cl}^{\mathrm{a}}$ \\
\hline \multicolumn{13}{|l|}{ Gender } \\
\hline Male & ref & & & & ref & & ref & & & & ref & \\
\hline Female & 0.61 & $(0.39,0.95)$ * & & & 0.97 & $(0.58,1.61)$ & 0.91 & $(0.66,1.24)$ & & & 1.14 & $(0.82,1.57)$ \\
\hline \multicolumn{13}{|l|}{ Age group } \\
\hline $51 \sim$ & ref & & & & ref & & ref & & & & ref & \\
\hline $18 \sim 30$ & 3.49 & $(1.91,6.39)$ & & & 2.77 & $(1.41,5.43)$ & 1.39 & $(0.91,2.13)$ & & & 1.48 & $(0.94,2.33)$ \\
\hline $31 \sim 40$ & 2.06 & $(1.13,3.77)$ * & & & 1.92 & $(0.96,3.82)$ & 0.90 & $(0.55,1.48)$ & & & 0.94 & $(0.56,1.58)$ \\
\hline $41 \sim 50$ & 1.54 & $(0.91,2.61)$ & & & 1.60 & $(0.88,2.81)$ & 1.05 & $(0.65,1.68)$ & & & 1.02 & $(0.62,1.67)$ \\
\hline \multicolumn{13}{|l|}{$\begin{array}{l}\text { Education } \\
\text { background }\end{array}$} \\
\hline $\begin{array}{l}\text { College and } \\
\text { above }\end{array}$ & ref & & & & ref & & & & & & & \\
\hline $\begin{array}{l}\text { Primary school } \\
\text { or below }\end{array}$ & 1.46 & $(0.74,2.88)$ & & & 0.67 & $(0.31,1.46)$ & - & - & & & - & - \\
\hline $\begin{array}{l}\text { Junior middle } \\
\text { school }\end{array}$ & 2.16 & $(1.25,3.73)^{\star \star}$ & & & 1.49 & $(0.80,2.76)$ & - & - & & & - & - \\
\hline High school & 1.37 & $(0.84,2.24)$ & & & 1.00 & $(0.57,1.76)$ & - & - & & & - & - \\
\hline \multicolumn{13}{|l|}{$\begin{array}{l}\text { Live together } \\
\text { with } \\
\text { Spouse/Partner }\end{array}$} \\
\hline No & ref & & & & ref & & & & & & & \\
\hline Yes & 0.93 & $(0.62,1.40)$ & & & 1.00 & $(0.64,1.58)$ & - & - & & & - & - \\
\hline \multicolumn{13}{|l|}{ Accommodation } \\
\hline $\begin{array}{l}\text { Self-renting } \\
\text { room/ Self- } \\
\text { purchased } \\
\text { house }\end{array}$ & ref & & & & ref & & & & & & & \\
\hline $\begin{array}{l}\text { Co-renting } \\
\text { room/ } \\
\text { Dormitory }\end{array}$ & 1.34 & $(0.90,2.01)$ & & & 1.36 & $(0.87,2.13)$ & - & - & & & - & - \\
\hline \multicolumn{13}{|l|}{ Type of work } \\
\hline $\begin{array}{l}\text { Secondary } \\
\text { industry }\end{array}$ & ref & & & & ref & & & & & & & \\
\hline Tertiary Industry & 1.08 & $(0.71,1.63)$ & & & 0.91 & $(0.56,1.49)$ & - & - & & & - & - \\
\hline \multicolumn{13}{|l|}{$\begin{array}{l}\text { Working hours } \\
\text { per day }\end{array}$} \\
\hline$\leq 8 \mathrm{~h}$ & ref & & & & ref & & ref & & & & ref & \\
\hline$>8 \mathrm{~h}$ & 1.25 & $(0.85,1.82)$ & & & 1.39 & $(0.90,2.13)$ & 1.22 & $(0.92,1.64)$ & & & 1.25 & $(0.93,1.69)$ \\
\hline \multicolumn{13}{|l|}{$\begin{array}{l}\text { Monthly } \\
\text { personal income } \\
\text { (RMB) }\end{array}$} \\
\hline$>4000$ & ref & & & & ref & & ref & & & & ref & \\
\hline$<2500$ & 0.92 & $(0.54,1.54)$ & & & 0.87 & $(0.49,1.55)$ & 0.86 & $(0.60,1.22)$ & & & 0.77 & $(0.54,1.12)$ \\
\hline $2501 \sim 4000$ & 1.16 & $(0.76,1.76)$ & & & 1.03 & $(0.65,1.63)$ & 0.94 & $(0.70,1.24)$ & & & 0.85 & $(0.63,1.14)$ \\
\hline
\end{tabular}

${ }^{\mathrm{a} O R}$ odds ratios; ${ }^{\mathrm{b}} \mathrm{Cl}$, confidence intervals; $* P<0.05$, ** $P<0.01$ *** $P<0.001$ 


\begin{tabular}{|c|c|c|c|c|c|c|c|c|c|c|c|c|}
\hline \multirow[t]{3}{*}{ Variables } & \multicolumn{6}{|c|}{ Intention towards HB-related behaviors } & \multicolumn{6}{|c|}{ HB-related behaviors } \\
\hline & \multicolumn{3}{|c|}{ Block \ } & \multicolumn{2}{|l|}{ Block \ } & \multicolumn{2}{|l|}{ Block 【 } & \multicolumn{2}{|l|}{ Block \ } & \multicolumn{2}{|l|}{ Block \ } & Block \ \\
\hline & $\begin{array}{l}\mathrm{OR} \\
\mathrm{a}\end{array}$ & $95 \% \mathrm{Cl}^{\mathrm{b}}$ & $\begin{array}{l}\mathrm{OR} \\
\mathrm{a}\end{array}$ & $95 \% \mathrm{Cl}^{\mathrm{b}}$ & OR & $95 \% \mathrm{Cl}^{\mathrm{a}}$ & $\begin{array}{l}\text { OR } \\
a\end{array}$ & $95 \% \mathrm{Cl}^{\mathrm{b}}$ & $\begin{array}{l}\text { OR } \\
a\end{array}$ & $95 \% \mathrm{Cl}^{\mathrm{b}}$ & OR & $95 \% \mathrm{Cl}^{\mathrm{a}}$ \\
\hline \multicolumn{13}{|l|}{ Do you smoke? } \\
\hline No & ref & & & & ref & & ref & & & & ref & \\
\hline Yes & 1.35 & $(0.77,2.38)$ & & & 1.09 & $(0.58,2.06)$ & 1.43 & $(0.99,2.03)$ & & & 1.32 & $(0.92,1.89)$ \\
\hline \multicolumn{13}{|l|}{ Do you drink? } \\
\hline No & ref & & & & ref & & ref & & & & ref & \\
\hline Yes & 1.58 & $(0.93,2.71)$ & & & 1.54 & $(0.84,2.83)$ & 1.63 & $\underset{* \star}{(1.18,2.26)}$ & & & 1.57 & $(1.12,2.19)$ \\
\hline \multicolumn{13}{|l|}{$\begin{array}{l}\text { Level of HB } \\
\text { knowledge }\end{array}$} \\
\hline Good (11 13) & ref & & & & ref & & & & & & & \\
\hline Medium $(8 \sim 10)$ & 2.01 & $(1.12,3.60)$ * & & & 1.65 & $(0.86,3.20)$ & - & - & & & - & - \\
\hline Poor $(0 \sim 7)$ & 2.30 & $(1.22,4 \cdot 33)$ * & & & 2.10 & $(1.03,4.28)$ & - & - & & & - & - \\
\hline \multicolumn{13}{|l|}{ TPB variables } \\
\hline$A B$ & & & 9.36 & $\underset{\star \star \star}{(5.32,16.46)}$ & 9.49 & $\underset{\star \star \star}{(5.32,16.91)}$ & & & 1.29 & $(1.02,1.63)$ & 1.27 & $(0.99,1.61)$ \\
\hline BI & & & - & - & - & - & & & 1.38 & $(1.07,1.76)$ & 1.42 & $(1.10,1.82)$ \\
\hline SN & & & 2.20 & $\underset{\star \star \star \star}{(1.54,3.17)}$ & 2.06 & $(1.44,2.95)$ & & & 1.19 & $(1.00,1.41)$ & 1.16 & $(0.97,1.38)$ \\
\hline PBC & & & 1.10 & $(0.98,1.24)$ & 0.99 & $(0.86,1.14)$ & & & 1.01 & $(0.92,1.11)$ & 0.95 & $(0.86,1.05)$ \\
\hline EB & & & 1.92 & $\underset{\star \star \star \star}{(1.43,2.58)}$ & 2.17 & ${ }_{\star \star \star}^{(1.60,2.94)}$ & & & 1.29 & $\underset{\star \star}{(1.07,1.56)}$ & 1.23 & ${ }_{\star}^{(1.01,1.50)}$ \\
\hline RF & & & 1.20 & $(1.05,1.38)$ & 1.23 & ${ }_{\star \star}^{(1.06,1.42)}$ & & & 1.10 & $(0.99,1.24)$ & 1.13 & ${ }_{\star}^{(1.02,1.25)}$ \\
\hline
\end{tabular}

\section{Influencing factors associated with HB-related risk behaviors}

As table 2shows, univariate analyses indicated that the risk behavior of respondents was significantly different with variables of gender, age, working hours per day, monthly personal income, smoking and alcohol consumption respectively $(P<0.05)$. Binary logistic regression detected thatsmoking $(\mathrm{OR}=1.43$,

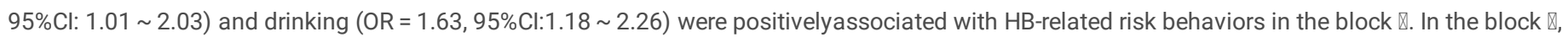
modules of $\mathrm{AB}(\mathrm{OR}=1.29,95 \% \mathrm{Cl}: 1.02 \sim 1.63), \mathrm{BI}(\mathrm{OR}=1.38,95 \% \mathrm{Cl}: 1.07 \sim 1.76)$ and $\mathrm{EB}(\mathrm{OR}=1.29,95 \% \mathrm{Cl}: 1.07 \sim 1.56)$ were positivelyassociated with risk

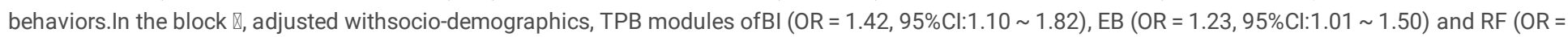
1.13, 95\%Cl:1.02 1.25), werepositivelyassociated with risk behaviors (Table 5).

\section{Discussion}

The Action Plan for Prevention and Treatment of Viral Hepatitis in China (2017-2020) has underlined the significance to prevent and control of viral hepatitis,particularly for those who are susceptible to the disease and possible to spread the transmission[27]. Rural-to-urban migrants may play a crucial role in the cross-regional diffusion of HBV in China due to their frequently seasonal moves over workplaces and homelands[13]. Existing evidence showed that the proportion of migrant workers who performed risk behaviors related to sexually transmitted diseases (STDs) were relatively high[13]. Our findings, verifying this point, detected that about one-third of participants had at least once of HB-related risk behaviors during in the last six months, although the proportion of migrant workers' causally extramarital sex $(10.24 \%)$ and commercial sex $(4.7 \%)$ were lower than those reported in studies conducted in Shanghai (15.22\%) and Zhejiang (5.7\%)[28].In addition, $2.80 \%$ of migrant workers had admitted to have homosexual behaviors, which reminds us to also pay attention because MSM have been referred to be highly risky for STDs[19].

Although the Chinese Ministry of Health has advocated condom use to prevent STD ssince 2006 [29],in our study a considerably big proportion of migrant workers (58.19\%)never wore a condom when having any types of sexual behaviors. This finding was in line with the high rate of condom nonuse for migrant 
workers in Hefei, China (52.68\%)[12].Regarding the potential barriers for condom use, having taken other contraception methods, uncomfortable to wear and no requirement by the partner took the top three positions. Those indicate that wearing a condom was only viewed as a contraception way but not understood as a protection from STDs by migrant workers, and the pleasant sensation outdid the perception of disease infection. All those have pointed to the weak knowledge and awareness of STDs among migrant workers, which is right consistent with the generally low level of HB knowledge for respondents in our study. In fact, protected sex with a condom has been proved to be significantly practical and cost-effective to prevent STD transmission[19],and therefore, extensive publicity for condom usage is in need to be strengthened among migrant workers. Meanwhile, vending machines for condoms (better for free) could be set near by migrant workers' living areas to cope with the embarrassed feeling mention by the respondents[30].

Similar as we though, there was a small part of respondents having a history of injector sharing for drug use (1.00\%) and illegal blood selling/transfusion $(1.23 \%)$, in line with studies with the migrant workers in eastern China[12, 28]. Blood transmission, a significant route of HBV infection as well as sexual transmission, should not be neglected. In addition, one-fifth of participants in our study were detected sharing personal hygiene products like toothbrushes and/or towels, which would amplifythe possibility of HBV infection through the broken skin[31]. Therefore, health educationtargeting those issues need to be enhanced with migrant workers.

Approximately one-third of migrant workers admitted having had HB-related risk behaviors,moreover, nearly $90 \%$ of respondents expressed that they would like to perform some risk behaviors sometime. This is a potential threat as behavioral intention indicates a possibility for a person to have actual behavior. Therefore, educational intervention on altering behavior intention and self-protection cognition is crucial in addition to directly regulating on risk behaviors.

Similar with previous studies, logistic regressions suggested that migrant workers who were male, at younger age, with lower educational background and at lower knowledge level would be more intended to act HB-related risk behaviors[17]. Compared with females, most males are less perceived with disease risk and overestimate their own health status[32]. Compared with older ones, young people are right in a sexually active period and will probably have more sexual demands[28]. Compared with higher educated ones, people with lower education tend to be less cognitive for disease prevention[33].Compared with individuals at a higher knowledge level, those with weak HB knowledge may lack understanding of HB and be less aware of self-protection against the disease[32]. Consistent with the study on HIV-related behaviors in northwest Ethiopia, there was a positive association between drinking and performing risk behaviors. It is to some extent because drinking probably results in more opportunities for casual sex and unprotected sex for migrant workers [34].

As adjusted by socio-demographics, migrant workers scored higher points of AB and SN were more intended to act risk behaviors, and those scored higher points of $\mathrm{BI}$ were more likely to have performed HB-related risk behaviors. All these can be exactly interpreted by the typical TPB framework - AB and SN, produced from behavioral beliefs and normative beliefs, will act on $\mathrm{BI}$, and then work together with $\mathrm{BI}$ to trigger the behavior[16]. That is, if migrant workers have a more favorable attitude towards HB-related risk behaviors and less perceived social pressure upon acting the behaviors, they would be more intended to do so, and also be more likely put into practice[15]

To strengthen the interpretability of the practical behaviors of migrant workers, two socio-psychological modules - EB and RF - were introduced into the typical TPB framework. As we expected, the two variables were positively associated with both behavioral intention and practical risk behaviors. Previous studies argued that daily decision making would be affected by the actual emotional experience, and successful implementing of risk behaviors in the past appears to render migrant workers more likely to repeat in the future[35]. Besides, the stronger regret feeling migrant workers have, the less intended they would be and also less likely to perform risk behaviors. It is because regret representing a negative consciousness and an emotional reaction to persons' intention or behaviors[17].Given migrant workers showed low perception and poor self-protection against HBV infection, health educational campaigns are necessary to improve their cognition and behaviors as mentioned above. Considering friends/family members, television/radio and internet /cell phone APPs are widely popular sources for migrant workers to gain health knowledge and information, peer education will work, and the health education through the combination of new and traditional media will be also encouraged. Besides, only half of migrant workers have inoculated HB vaccine in our study. It is partially because free HB immunization program is not offered to people aged above 15 currently in China[3]. Therefore, a sound compensation system is in expectation to provide extra financial support for HB-susceptible adults including migrant workers to expand the coverage of the HB vaccine.

There were some limitations necessary to be noticed. Firstly, causal inference based on the associations observed in our study might be more or less limited as it was in cross-sectional study design. Secondly, selection bias, giving rise to an imbalance of occupation distribution between the sampled participants and the whole population of Chongqing's migrant workers, might be introduced as it was non-random sampling. In addition, report bias, mostly assumed as an underestimate of acting risk behaviors, might be inevitable due to the personal privacy and social desirability, although anonymity of respondents was reassured.

\section{Conclusions}

There was one-third of migrant workers having had HB-related risk behaviors and nearly $90 \%$ of migrant workers intended to act risk behaviors in the present study. The Condom was poorly used among migrant workers for STDs prevention. Migrant workers who were male, less educated, poorer in HB knowledge and at a younger age have the stronger intention of HB-related risk behaviors, while those who were drinking alcohol were more likely to have performed risk behaviors. As completed with innovative variables of EB and RF, the TPB framework played well in interpreting the influencing factors, showing that migrant workers have a more positive attitude and less subject norm towards risk behaviors would be more intended and then be more likely to act. Accordingly, more attention should be paid to both improving the disease perception and self-protection awareness, as well as helping migrant workers to regulate their behaviors. Meanwhile, theory-grounded health educational interventions, targeting the key influencing factors proposed by the analyses, are in need of peer education and the combination of both new and traditional media.

\section{Declarations}


Ethic Approval and Consent to participate:

The present study was approved by Institutional Review Broad of CQMU (No. 2018016), and the informed consent was obtained from every respondents included in the study.

Consent for publication:

All of the authors have approved the manuscript and agreed to publish the study.

Availability of supporting data:

Not applicable.

Competing interests:

The authors declare no competing interests.

Funding:

The present study was funded by National Natural Science Foundation of China (No.71603034) and Medical Data Science Academy Project (No. ZHYX202022).

Authors' contributions:

$\mathrm{XL}$ and $\mathrm{XJT}$ conceived and designed the study. XL, HX, MX, ML, YT, XSS, DSW, KL, RC performed field surveys and data collection. HX, ML and XL conducted data analyses. HX, LMJ, and XL drafted the manuscript. XJT reviewed and polished the manuscript. All the authors have cautiously read and approved the final version of the manuscript.

Acknowledgements:

We would like to thank local health institutions and the Urban-rural Development Committee for their kind assistance and coordination throughout the field study. All the migrant workers who participated in the study are much appreciated.

\section{References}

1. Howell J, Van Gemert C, Lemoine M, Thursz M, Hellard M: Overview of hepatitis B prevalence, prevention, and management in the Pacific Islands and Territories. Journal of gastroenterology and hepatology 2014, 29(11):1854-1866.

2. World Health Organization. World Health Organization. Hepatitis B [http://www.who.int/news-room/fact-sheets/detail/hepatitis-b]

3. National Bureau of Disease Prevention and Control of China. The National Epidemiological Serosurvey Result of Hepatitis B [http://www.chinacdc.cn/dcbg/200804/t20080423_34870.htm]

4. Qiu Y, Ren J, Yao J: Healthy adult vaccination: An urgent need to prevent hepatitis B in China. Human vaccines \& immunotherapeutics 2016, 12(3):773778.

5. L Z, S R, W Z: On the sexual transmission dynamics of hepatitis B virus in China. Journal of theoretical biology 2015, 369(undefined):1-12.

6. A R, R A, JE V, A A, N Z, W M, M B, E M, A E, M S: HCV, HBV, and HIV seroprevalence, coinfections, and related behaviors among male injection drug users in Arak, Iran. AIDS care 2014, 26(9):1122-1126.

7. Martinson FE, Weigle KA, Royce RA, Weber DJ, Suchindran CM, Lemon SM: Risk factors for horizontal transmission of hepatitis B virus in a rural district in Ghana. American journal of epidemiology 1998, 147(5):478-487.

8. X C, IR R, T Y, R C: Work stress, life stress, and smoking among rural-urban migrant workers in China. BMC public health 2012,12 (undefined):979.

9. National Bureau of Statistics. Migrant Workers Monitoring Investigation Report of China in 2018. 2019 (In Chinese) [Available online: http://www.stats.gov.cn/tjsj/zxfb/201904/t20190429_1662268.html]

10. C Z, Q G, H Y, L C, X F, W J: Quality of life in China rural-to-urban female migrant factory workers: a before-and-after study. Health and quality of life outcomes 2013, 11(undefined):123.

11. Wei L, Zhou J, Tan Z, Li D, Wang Q: Survey on HIV / AIDS related prevention knowledge, attitudes and high-risk behavior among migrant workers in Chongqing. Chinese Journal of Family Planning 2010, 18(07):411-413

12. Xin Q, Huang Y, Hu C, Wang J, Zhang X, Zhang Z, Yang L, Huang F: High-risk behaviors and condom use among migrant workers at construction sites. Journal of Chinese Public Health 2010, 26(07):914

13. Zou X, Chow EP, Zhao P, Xu Y, Ling L, Zhang L: Rural-to-urban migrants are at high risk of sexually transmitted and viral hepatitis infections in China: a systematic review and meta-analysis. BMC infectious diseases 2014, 14:490.

14. Lau JT, Thomas J: Risk behaviours of Hong Kong male residents travelling to mainland China: a potential bridge population for HIV infection. AIDS Care 2001, 13(1):71-81.

15. Ajzen I, Fishbein M: Understanding attitudes and predicting social behavior. Englewood Cliffs NJ 1980.

16. Ajzen I: Constructing a TpB Questionnaire: Conceptual and Methodological Considerations. 2006.

Page 15/16 
17. CJ S, AS G, CK G, EA M, AD B, M C: Feel good now or regret it later? The respective roles of affective attitudes and anticipated affective reactions for explaining health-promoting and health risk behavioral intentions. Journal of applied social psychology 2019, 49(6):331-348.

18. JC L-J, A P-G, FJ O-R: Behavioral Intention to Gamble Among Adolescents: Differences Between Gamblers and Non-gamblers-Prevention Recommendations. Journal of gambling studies 2019.

19. Andrew BJ, Mullan BA, de Wit JB, Monds LA, Todd J, Kothe EJ: Does the Theory of Planned Behaviour Explain Condom Use Behaviour Among Men Who have Sex with Men? A Meta-analytic Review of the Literature. AIDS and behavior 2016, 20(12):2834-2844.

20. Abamecha F, Godesso A, Girma E: Intention to voluntary HIV counseling and testing (VCT) among health professionals in Jimma zone, Ethiopia: the theory of planned behavior (TPB) perspective. BMC public health 2013, 13:140.

21. Su X, Li L, Griffiths SM, Gao Y, Lau JT, Mo PK: Smoking behaviors and intentions among adolescents in rural China: the application of the Theory of Planned Behavior and the role of social influence. Addictive behaviors 2015, 48:44-51.

22. van Lettow B, de Vries H, Burdorf A, Conner M, van Empelen P: Explaining young adults' drinking behaviour within an augmented Theory of Planned Behaviour: temporal stability of drinker prototypes. British journal of health psychology 2015, 20(2):305-323.

23. Bureau of Statistics of Chongqing, China 2018. statistical yearbook. 2018 (In Chinese) [Available online: http://tjj.cq.gov.cn//tjnj/2018/indexch.htm]

24. Health commsssion of Chongqing,China. 2016, Health Statistic Yearbook. 2017 (In Chinese) [http://wsjkw.cq.gov.cn/sjfx/]

25. Gong.T., gong.Y., Deng.D., Ye.M.L.: A Cross-Sectional Study On Hepatitis B In Migrant Workers Of Chongqing (In Chinese). Modern Preventive Medicine 2007(20):3842-3844.

26. Health CMo: The national prevent and control plan of HB in China(2006 2010). Chinese Pratical Journal of Rural Doctors 2006(08):1-4.

27. Action plan for prevention and treatment of viral hepatitis in China(2017-2020) (In Chinese). Chinese Journal of Virology 2018, 8(01):1-5.

28. Pan X, Zhu Y, Wang Q, Zheng H, Chen X, Su J, Peng Z, Yu R, Wang N: Prevalence of HIV, syphilis, HCV and their high risk behaviors among migrant workers in eastern China. PloS one 2013, 8(2):e57258.

29. HIV Prevention and Control Regulations 2006 (In Chinese) [Available online: http://www.nhc.gov.cn/wjw/zcjd/201304/d46a4092080040c1bec7d7428ead4bd0.shtml]

30. Abstract on the Reply to the Proposal No. 0834 (Medical and Sports No. 094) of the Fourth Session of the 12th National Committee of the CPPCC. 2016 (In Chinese) [Available online: http://www.nhc.gov.cn/wjw/tia/201611/620feea7ddc54a418ff1a6c233bb0e2c.shtml]

31. Madiba TK, Nkambule NR, Kungoane T, Bhayat A: Knowledge and Practices Related to Hepatitis B Infection among Dental and Oral Hygiene Students at a University in Pretoria. Journal of International Society of Preventive \& Community Dentistry 2018, 8(3):200-204.

32. Liu C, Lvu YK: Analysis on hepatitis B related KAP among migrants in Beijing (In Chinese). Chinese Journal of Health Education 2015, 31(11):1029-1032.

33. A M, P V-M, D A-L, A S-R, P G-R, M G-L, E G-M, L M-B, J N-C, D P-P et al: Sexual risk among Colombian adolescents: knowledge, attitudes, normative beliefs, perceived control, intention, and sexual behavior. BMC public health 2018, 18(1):1377.

34. K T, B W, H G: Sexual behavior and vulnerability to HIV infection among seasonal migrant laborers in Metema district, northwest Ethiopia: a crosssectional study. BMC public health 2015, 15(undefined):122.

35. M P, J C: Sexual behavior and perceived risk of HIV/AIDS among young migrant factory workers in Nepal. The Journal of adolescent health : official publication of the Society for Adolescent Medicine 2006, 38(3):237-246. 